\title{
Confiabilidade e validade do BESTest e do MiniBESTest em hemiparéticos crônicos
}

\author{
Reliability and validity of the BESTest and MiniBESTest in chronic hemiparesis
}

Carla Bambirra1, Livia de Castro Magalhães², Fátima Rodrigues-de-Paula ${ }^{3}$

\section{RESUMO}

Objetivo. Avaliar a confiabilidade e validade das versões brasileiras do BESTest e MiniBESTest em hemiparéticos crônicos. Método. As confiabilidades teste-reteste e interexaminadores foram avaliadas pelo coeficiente kappa ponderado. A validade de construto foi analisada através do modelo Rasch em 40 hemiparéticos (58,8 \pm 12 ,8anos). Resultados. As confiabilidades teste-reteste e interexaminadores apresentaram concordância de moderada a quase perfeita para o BESTest $(0,48 \leq \mathrm{Kw} \leq 1,0 ; \mathrm{p}<0,05)$ e forte a quase perfeita para o MiniBESTest $(0,62 \leq \mathrm{Kw} \leq 1,0 ; \mathrm{p}<0,05)$. Os itens dos instrumentos dividiram as pessoas em, aproximadamente, três níveis de habilidade. A estabilidade das medidas dos indivíduos e dos itens variou de 0,89 a 0,96 para os instrumentos. No BESTest, o item mais difícil foi "sentar no chão e levantar" e no MiniBESTest "correção com passo lateral à direita". O item mais fácil para ambos foi "permanecer de olhos abertos, superfície firme". O nível de dificuldade dos itens dos instrumentos foi apropriado para o nível de habilidade dos indivíduos. Dois itens do BESTest apresentaram padrão errático. No MiniBESTest não houve item errático. Conclusóes. As versóes brasileiras do BESTest e do MiniBESTest são confiáveis e válidas para a avaliação do equilíbrio em hemiparéticos crônicos. Recomenda-se atenção quanto aos itens erráticos e cautela na interpretação do escore total do BESTest.

Unitermos. Equilíbrio Postural, Avaliação da Deficiência, Paresia, Acidente Vascular Cerebral, Psicometria

Citação. Bambirra C, Magalhães LC, Rodrigues-de-Paula F. Confiabilidade e validade do BESTest e do MiniBESTest em hemiparéticos crônicos.

Trabalho realizado na Universidade Federal de Minas Gerais, Belo Horizonte-MG, Brasil.

Suporte financeiro. CNPq e Fapemig

\begin{abstract}
Objective. To evaluate the reliability and construct validity of the Brazilian versions of the BESTest and MiniBESTest in chronic hemiparesis. Method. The test-retest and interrater reliability were assessed using the weighted kappa coefficient. The Rasch model was used for analysis of construct validity in 40 hemiparetic subjects (58.8 \pm 12.8 years). Results. Moderate to almost perfect agreement was observed for the BESTest for test-retest and interrater $(0.48 \leq \mathrm{Kw} \leq 1.0$; $\mathrm{p}<0.05)$. Strong to almost perfect agreement was observed for MiniBESTest for test-retest and interrater reliability $(0.62 \leq \mathrm{Kw} \leq 1.0$; $\mathrm{p}<0.05)$. The items of the BESTest and MiniBESTest divided people in about three skill levels. The individuals and items stability measurement ranged from 0.89 to 0.96 for the instruments. In BESTest, the most difficult item was "sit on the floor and stand up" and in MiniBESTest "compensatory stepping correction lateral to the right." The easier item for both instruments was "stand up on firm surface, eyes open." The level of difficulty of the items of both instruments was appropriate for the skill level of individuals. Two items of the BESTest were erratic. In MiniBESTest were not observed erratic items. Conclusion. The Brazilian versions of the BESTtest and MiniBESTest are reliable and have adequate construct validity to evaluate balance in chronic hemiparetic individuals. It is recommended, however, caution in interpreting the total score of the BESTest.
\end{abstract}

Keywords. Postural Balance, Disability Evaluation, Paresis, stroke, Psychometrics

Citation. Bambirra C, Magalhães LC, Rodrigues-de-Paula F. Reliability and validity of the BESTest and MiniBESTest in chronic hemiparesis. 


\section{INTRODUÇÃO}

O equilíbrio é um construto complexo que envolve a contribuição de vários sistemas fisiológicos ${ }^{1}$. As desordens do equilíbrio podem ser resultantes do envelhecimento ou de doenças, tais como o acidente vascular cerebral (AVC) ${ }^{2}$. Os déficits de equilíbrio nos indivíduos com hemiparesia decorrente de AVC estấo relacionados a alto risco de quedas3. As conseqüências das quedas após AVC envolvem limitação da atividade, restrição da participação social, aumento da dependência e desenvolvimento do medo de cair ${ }^{3,4}$.

A avaliação do equilíbrio é importante tanto para o diagnóstico das desordens do equilíbrio quanto para o planejamento de intervençôes ${ }^{1}$. Instrumentos utilizados para a avaliação do equilíbrio em indivíduos com hemiparesia, como a Escala de Equilíbrio de Berg e a Escala de Fugl-Meyer - seção equilíbrio, apresentam efeito teto e solo nesta população ${ }^{5,6}$, o que pode limitar a abrangência dos indivíduos avaliados e a acurácia da avaliação.

O Balance Evaluation Systems Test (BESTest) é um instrumento que tem como objetivo ajudar na identificação dos subsistemas do controle postural que podem ser responsáveis pela alteração do equilíbrio funcional para que o tratamento possa ser direcionado aos subsistemas mais envolvidos no déficit do equilíbrio ${ }^{1}$. O BESTest consiste de 36 tarefas organizadas em 27 itens, os quais são agrupados em seis seções: restrições biomecânicas, limites de estabilidade/verticalidade, ajustes posturais antecipatórios, respostas posturais reativas, orientação sensorial e estabilidade na marcha com e sem uma tarefa cognitiva. Cada item é pontuado em uma escala ordinal de quatro pontos: de zero (pior desempenho) a três (melhor desempenho) ${ }^{1}$. A validade concorrente foi avaliada pela correlação entre o BESTest e a Activities-specific Balance Confdence em indivíduos com condiçóes neurológicas diversas e indivíduos sem alterações neurológicas, com coeficiente de correlação de Spearman de 0,641.

Uma versão reduzida do BESTest (MiniBESTest) foi desenvolvida com o intuito de facilitar a utilização do instrumento na prática clínica ${ }^{7}$. O MiniBESTest requer um tempo de 10-15 minutos para ser administrado e apresentou excelentes propriedades psicométricas em indivíduos com diagnósticos neurológicos diversos ${ }^{7}$ e com doença de Parkinson ${ }^{8,9}$. Recentemente, o BESTest e o Mi-
niBESTest foram traduzidos e adaptados transculturalmente para o português-Brasil em um estudo com idosos e indivíduos com doença de Parkinson, apresentando evidência de adequada validade de construto e estabilidade das respostas nestas populaçóes ${ }^{10}$.

A confiabilidade de uma medida define sua reprodutibilidade e a validade de construto determina a habilidade do instrumento em medir um conceito abstrato $^{11}$. Na validação de novos instrumentos, há uma tendência crescente para a utilização do modelo $\operatorname{Rasch}^{7}$, por este modelo fornecer uma representação mais acurada do processo de medição ${ }^{12}$. Dessa forma, este estudo objetivou avaliar a confiabilidade e a validade do BESTest e do MiniBESTest em indivíduos com hemiparesia, utilizando o modelo Rasch para avaliação da validade de construto destes instrumentos.

\section{MÉTODO}

Estudo metodológico realizado no Centro de Reabilitação Noroeste na cidade de Belo Horizonte, Minas Gerais, Brasil, durante o período de abril a setembro de 2012. O projeto foi aprovado pelos comitês de Ética da Universidade Federal de Minas Gerais e da Secretaria Municipal de Saúde de Belo Horizonte (parecer no 0680.0.203.410-11).

\section{Amostra}

A amostra foi de conveniência, composta por indivíduos com hemiparesia crônica. O cálculo amostral baseou-se na recomendação de 10 indivíduos para cada categoria de escore do instrumento ${ }^{13-15}$, sendo necessários, dessa forma, 40 indivíduos para obter calibraçôes estáveis dos itens na avaliação do BESTest. Para o MiniBESTest, seriam necessários 30 indivíduos hemiparéticos.

Os participantes foram recrutados a partir de convite oral, sendo incluídos no estudo aqueles que atenderam aos seguintes critérios: diagnóstico médico prévio de AVC; tempo de evolução após AVC de, pelo menos, seis meses; idade igual ou superior a 20 anos; de ambos os sexos; com hemiparesia caracterizada por escore inferior a 30, em um total de 34 pontos, na escala de avaliação dos membros inferiores de Fugl-Meyer (EFM) ${ }^{16}$ e capazes de deambular seis metros sem assistência humana, com ou 
sem utilização de órtese ou dispositivo de auxílio.

Foram excluídos os indivíduos que apresentaram: afasia de compreensão, definida pela resposta inadequada ao comando: "levante seu braço não comprometido e abra sua mão não comprometida" ${ }^{17}$; outras doenças neurológicas; déficit visual não corrigido; alteraçôes músculo-esqueléticas não relacionadas ao quadro de AVC com presença de dor e/ou instabilidade clínica que contraindicasse a realização do protocolo proposto. Todos os participantes assinaram o Termo de Consentimento Livre e Esclarecido.

\section{Procedimento}

Dados antropométricos, clínicos e demográficos foram coletados por meio da aplicação de questionário previamente elaborado. Os indivíduos foram caracterizados quanto ao nível de atividade física segundo recomendações estabelecidas pelo Physical Activity Trends/ USA/1990-1998 ${ }^{18}$. A EFM foi utilizada para caracterizar o retorno da função motora dos membros inferiores ${ }^{19}$.

As versóes brasileiras do BESTest e do MiniBESTest foram aplicadas conforme recomendaçóes prévias ${ }^{10}$. Antes do início da coleta foi realizado o treinamento das examinadoras através das instruçôes padronizadas e do vídeo interativo e educacional BESTest DVD-ROM. O BESTest e o MiniBESTest foram pontuados simultaneamente de acordo com o critério de cada um dos instrumentos. Pequenas adaptações foram necessárias em relação ao posicionamento do membro superior mais afetado de alguns indivíduos, que não apresentavam movimentação ativa neste membro. Nos itens realizados normalmente com os braços cruzados (sentado para de pé, inclinação lateral e verticalidade) estes indivíduos mantinham a máo menos afetada segurando a mais afetada junto ao tronco e em itens realizados com as mãos na cintura, o membro superior plégico foi mantido em sua postura habitual. No item "levantar o braço" o membro superior plégico era posicionado apoiado no halter, de forma a acompanhar o movimento de elevação realizado pelo outro membro.

\section{Análise estatística}

Confiabilidade

Para o estudo da confiabilidade participaram um total de 20 hemiparéticos, sendo 10 indivíduos partici- pantes da análise da confiabilidade teste-reteste e $10 \mathrm{da}$ análise da confiabilidade interexaminadores. A confiabilidade teste-reteste foi realizada por meio da aplicação do BESTest e MiniBESTest pelo mesmo avaliador, por duas vezes, num intervalo de sete dias. A confiabilidade interexaminadores foi realizada por meio da aplicação do BESTest e do MiniBESTest por duas examinadoras, as quais alternaram as instruçốes ao participante e observaram simultaneamente. Não houve nenhum tipo de comunicação entre as observadoras durante a pontuação dos instrumentos, de forma que uma examinadora não tinha conhecimento da pontuação da outra.

Como o BESTest é composto por uma escala ordinal com mais de três categorias de resposta foi utilizado o coeficiente Kappa ponderado para medida de confiabilidade teste-reteste e interexaminadores, sendo realizada análise para cada item ${ }^{20}$. O coeficiente Kappa ponderado também foi utilizado para as medidas de confiabilidade do MiniBESTest. A interpretação dos resultados do Kappa ponderado foi baseada nos seguintes pontos de corte: $\leq 0$ = concordância pobre; 0,01 a $0,20=$ fraca; 0,21 a $0,40=$ razoável; 0,41 a $0,60=$ moderada; 0,61 a $0,80=$ forte; $\mathrm{e}$ 0,81 a $1,0=$ quase perfeita ${ }^{20}$. O pacote estatístico Stats $D i-$ rect, versão 2.7.2 foi utilizado para a análise das confiabilidades.

\section{Análise Rasch}

As versôes brasileiras do BESTest e do MiniBESTest foram avaliadas com o uso do modelo Rasch, por meio do programa computadorizado específico Winsteps, versão 3.74.0/2012 21 . O modelo Rasch é um modelo probabilístico baseado no princípio de que quanto mais hábil é a pessoa, mais chances ela terá de obter sucesso em qualquer item, e quanto mais difícil o item, menores são as chances de qualquer pessoa obter sucesso ${ }^{12}$. No modelo Rasch, quando os itens de um instrumento medem o mesmo construto, é possível alinhar a dificuldade dos itens e o nível de habilidade dos indivíduos em um contínuo linear simples, dividido em intervalos iguais (logits) que podem ser utilizados para medir a habilidade das pessoas naquela dimensão ${ }^{12,22}$. No presente estudo, ao se avaliar o equilíbrio através do BESTest e do MiniBESTest, espera-se que quanto melhor a habilidade de equilíbrio do indivíduo, maior a probabilidade do mesmo em rece- 
ber escores altos em todos os itens das escalas. Por outro lado, quanto mais fácil o item, maior a probabilidade de qualquer pessoa receber escore alto no item.

Quando os indivíduos e os itens podem ser calibrados em um mesmo contínuo, o modelo permite determinar se o nível de dificuldade dos itens é apropriado para o nível de habilidade dos indivíduos. Além disso, os itens devem ser distribuídos de forma que cubram toda a variação de habilidade de equilíbrio apresentada pelos indivíduos, sem a presença de efeito teto e solo ${ }^{12}$.

O programa Winsteps oferece parâmetros como o $\mathrm{MnSq}$ (goodness-of-fit) e o valor z, que expressam a relação entre o escore esperado e o escore obtido. Se a relação está de acordo com os pressupostos do modelo, o MnSq tem valor igual a 1,0 , com variação de $\pm 0,3$ ou $\pm 0,412$. Valor muito alto de $\mathrm{MnSq}(>1,3)$ acompanhado por valor $\mathrm{z}>$ 2,0 , sinaliza itens erráticos ou que não medem a mesma dimensão. Valores de MnSq muito baixos $(<0,7)$ associados a $\mathrm{z}<-2,0$ sugerem pouca variabilidade de escores $\mathrm{e}$ indicam que o item tem pouco potencial para discriminar diferentes níveis de habilidade ${ }^{12}$. Os itens erráticos são aqueles em que, inesperadamente, pessoas com pior habilidade receberam escores altos ou vice-versa. Como o escore errático indica maior problema na definição do item, que pode comprometer o conceito de construto unidimensional, foram assinalados apenas os itens com valores de Mnsq altos em seus dois formatos, Infit e Outfit, que sinalizam flutuaçôes nas pontuações próximas ao nível de habilidade do indivíduo ou do nível de dificuldade do item e a presença de escores extremos, respectivamen$\mathrm{te}^{12,15}$. A literatura sugere que quando cerca de mais de $5 \%$ do número total de itens não se enquadram no modelo, isso é uma indicação de que os itens da escala não combinam para medir um conceito unidimensional ${ }^{15}$. Caso contrário, pode-se inferir que os itens em conjunto colaboram para definir uma dimensão ou construto ${ }^{10,15}$. Como o BESTest tem 36 tarefas, espera-se que não mais de 1,8 ou, arredondando, dois itens não se enquadrem no modelo. Para o MiniBESTest, que contem 16 tarefas, espera-se que não mais de 0,8 , ou um item, não se enquadre no modelo. $\mathrm{O}$ mesmo raciocínio se aplica ao padrão de respostas dos indivíduos avaliados. Outro dado a ser considerado é o resultado de análise fatorial (componente principal), incluída no programa Winsteps. A variância explicada pelas medidas $>50 \%$ e eigenvalue da variância do primeiro resíduo $<2$ dão suporte a unidimensionalidade da escala ${ }^{22}$.

O modelo Rasch também reporta o erro associado à calibração dos itens e dos indivíduos, que informa a precisão das medidas obtidas. A partir dessa margem de erro obtém-se o "índice de separação" que é utilizado para estimar em quantos níveis de habilidade os itens dividem a amostragem, o que pode ser calculado com uso da fórmula: $H=(4 G+1) / 3$, onde $H=$ número de estratos e $G$ $=$ índice de separação ${ }^{12}$. Espera-se que um teste divida os participantes em pelo menos três níveis de habilidade ${ }^{12}$. Outros índices relacionados são os coeficientes de confiabilidade das medidas das pessoas e da calibração dos itens, os quais fornecem o grau de consistência ou estabilidade das estimativas, com variação de zero a um $^{12}$. Os coeficientes maiores que 0,80 são considerados bons e maiores que 0,90 excelentes ${ }^{7}$.

\section{RESULTADOS}

A Tabela 1 apresenta as características antropométricas, demográficas e clínicas da amostra. Participaram do estudo 40 indivíduos com hemiparesia crônica. Destes, 18 indivíduos (45\%) faziam uso de bengala (canadense ou simples), 35\% eram casados, $10 \%$ viúvos, $17,5 \%$ divorciados ou separados e $37,5 \%$ solteiros. Um indivíduo era analfabeto, 18 indivíduos tinham até quatro anos de estudo, 11 estudaram de cinco a oito anos, sete concluíram o ensino médio e três concluíram o ensino superior.

\section{Confiabilidade}

Os valores do coeficiente kappa ponderado quadrático foram adequados para ambos os instrumentos e estão descritos na Tabela 2. Foi observada concordância de moderada a quase perfeita para o BESTest e de forte a quase perfeita para o MiniBESTest, tanto para a confiabilidade teste-reteste quanto para a interexaminadores.

\section{Análise Rasch}

No BESTest, o valor do coeficiente de confiabilidade da medida dos indivíduos foi 0,94 e a estimativa da estabilidade de calibraçấo dos itens foi de 0,96 . No 
Tabela 1. Média, desvio padrão ou distribuição de frequência (\%) das características dos participantes do estudo $(\mathrm{n}=40)$.

\begin{tabular}{|c|c|}
\hline Variável & Valor \\
\hline Idade (anos) & $58,8 \pm 12,8(26$ a 87$)$ \\
\hline \multicolumn{2}{|l|}{ Sexo } \\
\hline Masculino & $21(52,5 \%)$ \\
\hline Feminino & $19(47,5 \%)$ \\
\hline IMC & $25,8 \pm 4,3(15,2$ a 35,5$)$ \\
\hline $\begin{array}{c}\text { Escore na escala de membros } \\
\text { inferiores da EFM }\end{array}$ & $20,7 \pm 7,8(7$ a 30$)$ \\
\hline \multicolumn{2}{|l|}{ Nível de atividade física } \\
\hline Vigoroso & 0 \\
\hline Moderado & $5(12,5 \%)$ \\
\hline Insuficiente & $3(7,5 \%)$ \\
\hline Inativo & $32(80 \%)$ \\
\hline $\begin{array}{l}\text { Tempo de evoluçáo pós-AVC } \\
\text { (meses) }\end{array}$ & $80,6 \pm 76,6$ (6 a 399) \\
\hline \multicolumn{2}{|l|}{ Tipo de AVC } \\
\hline Hemorrágico & $10(25 \%)$ \\
\hline Isquêmico & $29(72,5 \%)$ \\
\hline Náo informado & $1(2,5 \%)$ \\
\hline \multicolumn{2}{|l|}{ Hemiparesia } \\
\hline Direita & $23(57,5 \%)$ \\
\hline Esquerda & $17(42,5 \%)$ \\
\hline
\end{tabular}

IMC = Índice de massa corporal; EFM = Escala dos membros inferiores de Fugl-Meyer; AVC = Acidente vascular cerebral.

MiniBESTest, o valor do coeficiente de confiabilidade das medidas dos indivíduos foi 0,89 e da calibração dos itens foi 0,94 . O índice de separação dos indivíduos foi de 4,10 para o BESTest e de 2,91 para o MiniBESTest, indicando que os itens dividiram as pessoas em 5,8 e 4,21 níveis de habilidade, respectivamente. $\mathrm{O}$ índice de separação dos itens foi 5,16 no BESTest e 4,01 no MiniBESTest, dividindo os itens do BESTest em 7,21 níveis de dificuldade e do MiniBESTest em 5,68 níveis de dificuldade.

A calibração dos itens para o BESTest e o MiniBESTest está apresentada em ordem decrescente de dificuldade nas Tabelas 3 e 4, respectivamente. Os itens do BESTest cobrem 4,97 logits, a medida média dos indiví- duos ficou 0,39 logits acima da média dos itens e o item mais difícil foi "sentar no chão e levantar". No MiniBESTest os itens cobrem 9,07 logits, a medida média dos pacientes ficou apenas 0,17 logits abaixo da média dos itens e "correção com passo lateral à direita" foi o item mais difícil. O item mais fácil para ambos os instrumentos foi "permanecer de olhos abertos em superfície firme". Em geral, as seções do BESTest apresentaram combinação de itens, distribuídos ao longo do continuo de equilíbrio, com diferentes níveis de dificuldade.

Dos 36 itens do BESTest, dois foram erráticos (não se encaixaram nas expectativas do modelo): "força lateral de quadril e tronco" e "alcance funcional lateral à esquerda". Somente um indivíduo apresentou padrão errático de resposta no BESTest. No MiniBESTest não foram observados itens nem indivíduos com padrão errático.

A análise fatorial do BESTest indica que, embora a variância explicada pelas medidas seja de $56,5 \%$, o eigenvalue do primeiro resíduo é de 3,9, o que indica que o teste tem mais de uma dimensão. Foi feita análise subsequente das subescalas, mas nenhuma delas apresentou qualidades psicométricas adequadas como escala independente. No MiniBESTest, a variância explicada pelas medidas foi de 53,5\% e o eigenvalue de 3,3. A análise subsequente indicou que, embora seja possível criar uma escala unidimensional com apenas 11 itens, isso excluiria itens que tem relevância clinica ("sentado para de pé” e os itens de correção com passo compensatório à frente, para trás, lateral à direita e à esquerda).

A Figura 1 representa os contínuos do nível de dificuldade dos itens e de habilidade dos indivíduos do BESTest e do MiniBESTest. Não foram observados efeitos teto ou solo em ambos os instrumentos.

Tabela 2. Confiabilidade teste-reteste e interexaminadores dos instrumentos analisados.

\begin{tabular}{|c|c|c|}
\hline \multirow[t]{2}{*}{ Instrumentos } & \multicolumn{2}{|c|}{ Confiabilidade } \\
\hline & Teste-reteste & Interexaminadores \\
\hline BESTest & $0,55 \leq \mathrm{K}_{\mathrm{w}} \leq 1,0^{*}$ & $0,48 \leq \mathrm{K}_{\mathrm{w}} \leq 1,0^{*}$ \\
\hline MiniBESTest & $0,62 \leq \mathrm{K}_{\mathrm{w}} \leq 1,0^{*}$ & $0,71 \leq \mathrm{K}_{\mathrm{w}} \leq 1,0^{*}$ \\
\hline
\end{tabular}


Tabela 3. Calibração dos itens do BESTest.

\begin{tabular}{|c|c|c|c|c|c|c|c|}
\hline \multirow[t]{2}{*}{ Item } & \multirow{2}{*}{$\begin{array}{c}\text { Seçóes } \\
\text { do teste }\end{array}$} & \multirow[t]{2}{*}{ Calibraçáo } & \multirow[t]{2}{*}{ Erro } & \multicolumn{2}{|c|}{ Infit } & \multicolumn{2}{|c|}{ Outfit } \\
\hline & & & & $\mathrm{MnSq}$ & $\mathrm{Z}$ & MnSq & $\mathrm{Z}$ \\
\hline 5) Sentar no cháo e levantar & $\mathrm{RB}$ & 1,52 & 0,21 & 1,46 & 2,0 & 1,26 & 0,9 \\
\hline 11.a) De pé em uma perna - E & TR & 1,48 & 0,2 & 0,76 & $-1,2$ & 1 & 0,1 \\
\hline 11.b) De pé em uma perna - D & TR & 1,48 & 0,2 & 1,16 & 0,8 & 1,87 & 2,5 \\
\hline 4) Força lateral de quadril e tronco* & $\mathrm{RB}$ & 1,43 & 0,2 & 1,49 & 2,2 & 2,95 & 4,8 \\
\hline 18.b) Correçáo com passo lateral - D & $\mathrm{RR}$ & 1,23 & 0,2 & 1,34 & 1,6 & 1,13 & 0,6 \\
\hline 25) Passar sobre obstáculos & EM & 1,15 & 0,2 & 0,67 & $-1,8$ & 0,63 & $-1,6$ \\
\hline 12) Tocar degrau alternadamente & TR & 1,11 & 0,2 & 0,39 & -4 & 0,38 & $-3,2$ \\
\hline 18.a) Correçáo com passo lateral - E & $\mathrm{RR}$ & 1,07 & 0,2 & 1,24 & 1,2 & 1,08 & 0,4 \\
\hline 2) Alinhamento do centro de massa & $\mathrm{RB}$ & 0,96 & 0,2 & 0,89 & $-0,5$ & 0,8 & $-0,8$ \\
\hline 17) Correção com passo para trás & $\mathrm{RR}$ & 0,92 & 0,2 & 1,27 & 1,3 & 1,17 & 0,8 \\
\hline 27)TUG dupla tarefa & EM & 0,92 & 0,2 & 0,54 & $-2,7$ & 0,55 & $-2,2$ \\
\hline 3) Força e amplitude de tornozelo & $\mathrm{RB}$ & 0,88 & 0,2 & 0,59 & $-2,3$ & 0,66 & $-1,6$ \\
\hline 15) Resposta no lugar para trás & $\mathrm{RR}$ & 0,84 & 0,2 & 1,48 & 2,1 & 1,39 & 1,6 \\
\hline 16) Correção com passo compensatório & $\mathrm{RR}$ & 0,84 & 0,2 & 1,37 & 1,7 & 1,21 & 0,9 \\
\hline 19.d) Olhos fechados, espuma & OS & 0,65 & 0,2 & 0,80 & -1 & 0,89 & $-0,4$ \\
\hline 10) Ficar na ponta dos pés & $\mathrm{TR}$ & 0,57 & 0,2 & 0,71 & $-1,5$ & 0,71 & $-1,3$ \\
\hline 24) Andar e girar sobre o eixo & EM & 0,38 & 0,2 & 0,59 & $-2,3$ & 0,55 & $-2,3$ \\
\hline 26) TUG & EM & 0,30 & 0,2 & 0,39 & $-3,8$ & 0,37 & $-3,7$ \\
\hline 8.b) Alcance funcional lateral - D & LE & 0,22 & 0,2 & 1,38 & 1,7 & 1,34 & 1,5 \\
\hline 21) Marcha em superfície plana & EM & 0,18 & 0,2 & 0,25 & $-5,2$ & 0,29 & $-4,4$ \\
\hline 7) Alcance funcional para frente & LE & 0,14 & 0,2 & 0,73 & $-1,3$ & 0,77 & -1 \\
\hline 8.a) Alcance funcional lateral - $\mathrm{E}^{*}$ & LE & 0,14 & 0,2 & 1,5 & 2,1 & 1,54 & 2,1 \\
\hline 23) Andar com viradas de cabeça & EM & $-0,12$ & 0,2 & 0,78 & $-1,0$ & 0,82 & $-0,7$ \\
\hline 22) Mudança na velocidade da marcha & EM & $-0,25$ & 0,21 & 0,9 & $-0,4$ & 0,93 & $-0,2$ \\
\hline 14) Resposta no lugar para frente & $\mathrm{RR}$ & $-0,29$ & 0,21 & 1,13 & 0,6 & 1,19 & 0,8 \\
\hline 1) Base de apoio & $\mathrm{RB}$ & $-0,38$ & 0,21 & 1,47 & 1,9 & 1,67 & 2,4 \\
\hline 6.b) Inclinação lateral - D & LE & $-0,57$ & 0,21 & 0,67 & $-1,6$ & 0,77 & $-0,9$ \\
\hline 6.a) Inclinaçáo lateral - E & LE & $-0,60$ & 0,22 & 1,35 & 1,4 & 1,32 & 1,3 \\
\hline 19.c) Olhos abertos, espuma & OS & $-0,83$ & 0,22 & 1,40 & 1,6 & 1,20 & 0,8 \\
\hline 20) Inclinação, olhos fechados & OS & $-1,18$ & 0,23 & 1,14 & 0,6 & 1,77 & 2,4 \\
\hline 13) Levantar o braço & $\mathrm{TR}$ & $-1,67$ & 0,25 & 1,36 & 1,2 & 0,96 & 0 \\
\hline 19.b)Olhos fechados superfície firme & OS & $-1,75$ & 0,28 & 0,9 & $-0,2$ & 1,07 & 0,3 \\
\hline 6.d) Verticalidade sentado - D & LE & $-2,25$ & 0,29 & 1,25 & 0,8 & 1,42 & 1 \\
\hline 9) Sentado para de pé & TR & $-2,25$ & 0,34 & 1,25 & 0,8 & 0,74 & $-0,5$ \\
\hline 6.c)Verticalidade sentado - E & LE & $-2,8$ & 0,41 & 1,21 & 0,6 & 1,02 & $-0,2$ \\
\hline 19.a) Olhos abertos superfície firme & OS & $-3,45$ & 0,53 & 0,8 & $-0,3$ & 0,75 & $-0,1$ \\
\hline
\end{tabular}

*Itens erráticos $\mathrm{MnSq}>1,3$ e Z > 2. D = direita; E= esquerda. TUG = "Timed up and go"; RB = Restriçốes biomecânicas; TR = Transiçóes-Ajustes posturais antecipatórios; $\mathrm{RR}=$ Respostas posturais reativas; $\mathrm{EM}=$ Estabilidade na marcha; $\mathrm{OS}=$ Orientaçáo sensorial; $\mathrm{LE}=$ Limites de estabilidade-Verticalidade. 
Tabela 4. Calibração dos itens do MINIBESTest.

\begin{tabular}{|c|c|c|c|c|c|c|}
\hline \multirow[t]{2}{*}{ Item } & \multirow[t]{2}{*}{ Calibraçáo } & \multirow[t]{2}{*}{ Erro } & \multicolumn{2}{|c|}{ Infit } & \multicolumn{2}{|c|}{ Outfit } \\
\hline & & & $\mathrm{MnSq}$ & $\mathrm{Z}$ & MnSq & $\mathbf{Z}$ \\
\hline 6.b) Correçáo com passo lateral - D & 1,15 & 0,29 & 1,22 & 1 & 0,96 & 0,1 \\
\hline 3.a) De pé em uma perna - E & 1,06 & 0,29 & 0,51 & $-2,8$ & 0,76 & 0,98 \\
\hline 3.b) De pé em uma perna - D & 0,98 & 0,29 & 1,13 & 0,7 & 1,44 & 1,1 \\
\hline 6.a) Correçáo com passo lateral - E & 0,81 & 0,29 & 0,99 & 0 & 0,83 & $-0,3$ \\
\hline 13) Passar sobre obstáculos & 0,81 & 0,29 & 0,65 & $-1,8$ & 0,63 & -1 \\
\hline 2) Ficar na ponta dos pés & 0,73 & 0,28 & 1,34 & 1,6 & 1,13 & 0,5 \\
\hline 5) Correçáo com passo para trás & 0,73 & 0,28 & 1,11 & 0,6 & 0,99 & 0,1 \\
\hline 12) Andar e girar sobre o eixo & 0,65 & 0,28 & 0,73 & $-1,4$ & 0,69 & $-0,8$ \\
\hline 14)TUG dupla tarefa & 0,57 & 0,28 & 0,63 & -2 & 0,64 & -1 \\
\hline 4) Correçáo com passo compensatório & 0,49 & 0,28 & 1,49 & 2 & 1 & 1 \\
\hline 11) Andar com viradas de cabeça & $-0,21$ & 0,28 & 1,12 & 0,6 & 1,13 & 0,6 \\
\hline 8) Olhos fechados, espuma & $-1,13$ & 0,3 & 1,01 & 0,1 & 0,97 & 0 \\
\hline 10) Mudança na velocidade da marcha & $-1,22$ & 0,3 & 1,15 & 0,7 & 1,29 & 1,1 \\
\hline 9) Inclinaçáo, olhos fechados & $-2,27$ & 0,35 & 1,05 & 0,3 & 1,26 & 0,7 \\
\hline 1) Sentado para de pé & $-3,17$ & 0,42 & 0,78 & $-0,6$ & 0,42 & -1 \\
\hline 7) Olhos abertos superfície firme & $-7,92$ & 1.88 & & Medid & ínima & \\
\hline
\end{tabular}

$\mathrm{D}=$ direita; $\mathrm{E}=$ esquerda. $\mathrm{TUG}$ = "Timed up and go".

\section{DISCUSSÃO}

O presente estudo é o primeiro a investigar as propriedades do BESTest em indivíduos com hemiparesia decorrente de AVC utilizando o modelo Rasch. A versão original do BESTest já foi aplicada em indivíduos com condiçôes neurológicas diversas ${ }^{1}$, em indivíduos com doença de Parkinson ${ }^{8,23}$, fibromialgia ${ }^{24}$ e esclerose múlti$\mathrm{pla}^{25}$. Os dados deste estudo forneceram evidência quanto à confiabilidade e a validade de construto do BESTest $\mathrm{e}$ do MiniBESTest em indivíduos com hemiparesia crônica.

$\mathrm{Na}$ análise da confiabilidade, foram observados valores adequados do coeficiente Kappa ponderado para todos os itens dos instrumentos. O número de categorias de resposta em um instrumento afeta o coeficiente Kappa ponderado, dado que este coeficiente penaliza de forma diferenciada, considerando a magnitude da discordância observada ${ }^{20}$. Sendo assim, a maior variação observada para a concordância no BESTest (moderada a quase perfeita) em relação ao MiniBESTest (forte a quase perfeita) pode ser explicada pela presença de uma categoria de resposta a mais no BESTest. Estudos prévios, com outras populaçóes, avaliaram a confiabilidade interexaminadores da versão original do BESTest ${ }^{1,8,9}$ e do MiniBESTest ${ }^{8}$ por meio do coeficiente de correlaçáo intraclasse (CCI), sendo observados valores altos de CCI para ambos os instrumentos. A confiabilidade teste-reteste foi avaliada para as versóes brasileiras dos instrumentos, também por meio do CCI (do escore total), em idosos e indivíduos com doença de Parkinson, com valores considerados quase perfeitos ${ }^{10}$.

Os índices de confiabilidade do padrão de respostas da análise Rasch para os instrumentos também foram altos, considerados de bons a excelentes, o que significa que as respostas dos indivíduos podem ser reproduzidas em aplicaçóes subsequentes dos testes ${ }^{12}$. Os instrumentos também cumpriram a expectativa de dividir os indivíduos em pelo menos três níveis de habilidade e os itens em pelo menos dois níveis de dificuldade ${ }^{12}$.

Quanto ao nível de dificuldade dos itens dos instrumentos em relação ao nível de habilidade da amostra, 
Figura 1. Mapa representativo da distribuição dos indivíduos e itens no contínuo de equilíbrio, medido pelo BESTest (A) e pelo MiniBESTest (B).
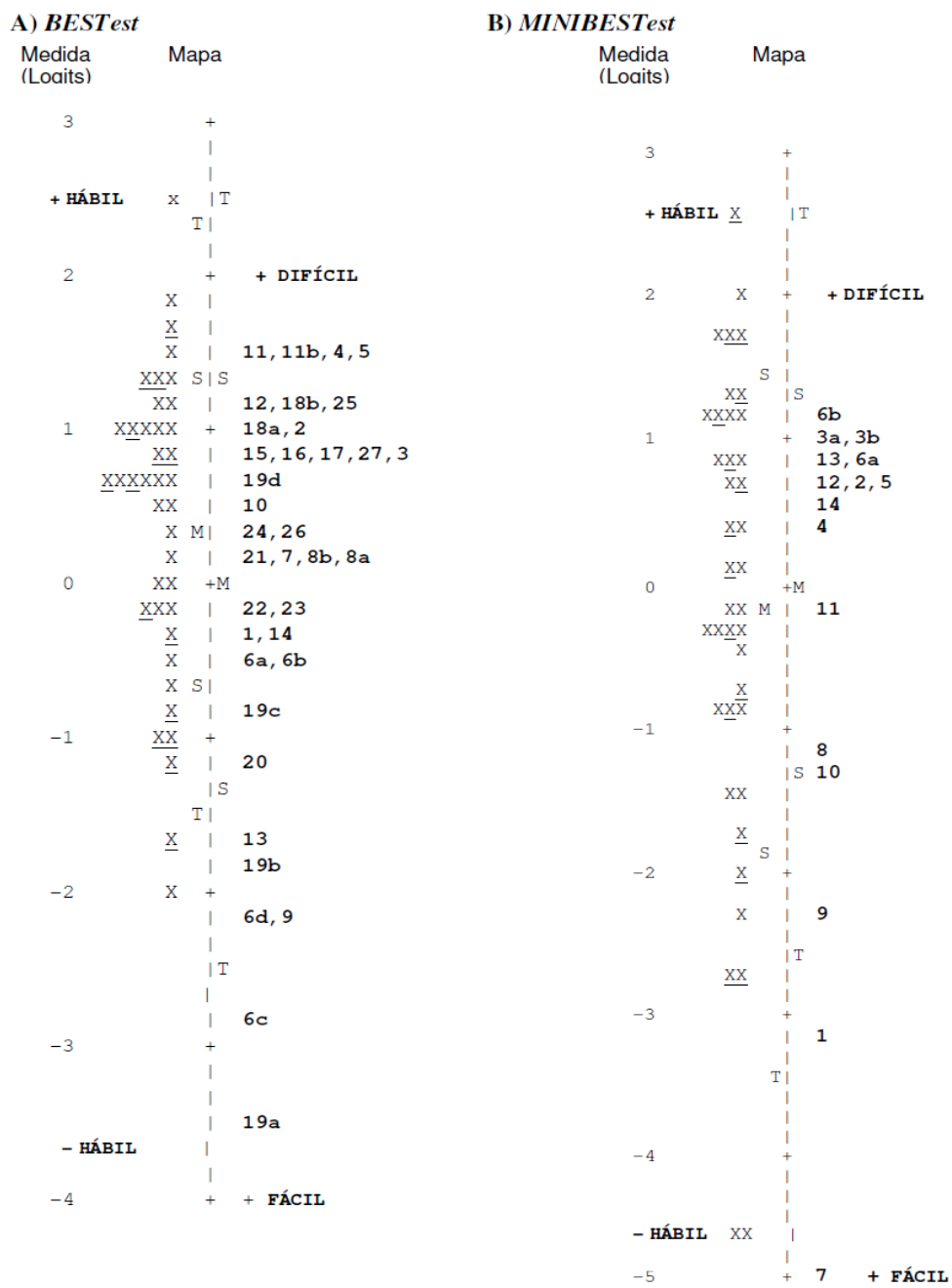

Cada $\mathrm{X}$ representa um indivíduo, sublinhados indicam idade $\geq 60$ anos. Ao longo da linha central: $\mathrm{M}=$ média; $\mathrm{S}=$ desvio padrăo; $\mathrm{T}=2$ desvios padrão. Os números à direita do contínuo representam os itens em cada um dos instrumentos.

percebe-se a presença de indivíduos no topo do contínuo sem itens alinhados (Figura 1). Entretanto, não houve efeito teto e as médias de calibração dos indivíduos ficaram bem próximas da média de dificuldade dos itens, o que sugere que os itens dos testes estão adequados para medir o desempenho da amostra, abrangendo todo o contínuo de habilidade dos indivíduos com hemiparesia crônica. A presença de vários itens do BESTest em um mesmo nível de dificuldade pode ser justificada por serem pertencentes a diferentes seçóes do instrumento. Os itens muito fáceis, observados na parte inferior da Figura 1, constituem uma vantagem, pois permitem que os testes possam ser utilizados em indivíduos mais debilitados.

Em relação à hierarquia da dificuldade dos itens (Tabela 3), o item mais difícil para os hemiparéticos no BESTest foi "sentar no chão e levantar", item não incluído no MiniBESTest. Não foram identificados estudos que fornecessem informaçôes específicas quanto à biomecânica do movimento de sentar no chão e levantar em indivíduos com hemiparesia. Entretanto, sabe-se que esta é uma atividade de grande demanda mecânica. Levantar-se do chão foi a limitação mais frequente observada 
em um estudo realizado com indivíduos com desordens musculo-esqueléticas diversas, como osteoartrite e lombalgia $^{26}$, o que demonstra que esta é uma tarefa difícil não só para indivíduos com hemiparesia. $\mathrm{O}$ item mais difícil no MiniBESTest (Tabela 4) foi "correção com passo lateral à direita", seguido pelos itens de apoio unipodal à esquerda e à direita e pelo item "correção com passo lateral à esquerda". Os itens referentes ao apoio unipodal e de correção com passo lateral também ficaram entre os mais difíceis no BESTest. A assimetria funcional presente na hemiparesia representa um desafio para o controle dos passos compensatórios ${ }^{27}$. Indivíduos com hemiparesia são geralmente relutantes em iniciar passos compensatórios com o membro parético ${ }^{27}$. Em um estudo realizado com hemiparéticos, quando o membro inferior preferencialmente utilizado para a resposta do passo foi bloqueado foram observadas respostas não apropriadas em $21 \%$ das tentativas, demonstrando que os indivíduos com hemiparesia apresentam limitada capacidade de adaptação nas respostas com passo compensatório ${ }^{27}$. Atividades que envolvem apoio unipodal também são desafiadoras ao equilíbrio devido à diminuição da base de suportel e à frequente incapacidade do membro parético em controlar o equilíbrio na postura de pé $e^{28}$. Inversamente, "permanecer de olhos abertos em superfície firme” foi o item mais fácil tanto no BESTest quanto no MiniBESTest, sendo uma atividade que envolve menores demandas de controle postural $^{1}$. De uma forma geral, a hierarquia e a adequação dos itens derivada da análise Rasch forneceu evidência quanto à validade de construto para ambos os instrumentos.

Comportamento errático foi detectado em dois itens do BESTest (item no. 4 e 8a), estando dentro do limite esperado para o instrumento. Ao examinar as pontuaçóes do item "força lateral de quadril e tronco", observou-se que três indivíduos com pobre habilidade de equilíbrio obtiveram escore correspondente a comprometimento leve neste item. O escore recebido equivale a conseguir abduzir ambos os quadris para levantar o pé do chão durante dez segundos, com apoio nas mãos do examinador, sem conseguir manter o tronco na vertical. Um aspecto que pode ter contribuído para tal resposta inesperada é o fato dos participantes deste estudo terem comprometimento crônico, e, possivelmente, apresentarem estratégias compensatórias bem desenvolvidas. No item "alcance funcional lateral à esquerda" observou-se resposta inesperada em um indivíduo que, apesar de ter uma alta habilidade de equilíbrio, recebeu pontuação igual a zero neste item. Este indivíduo não apresentava movimentação ativa no membro superior esquerdo, não sendo capaz de realizar o alcance lateral. Neste caso, um fator não relacionado diretamente ao controle postural influenciou a resposta. O padrão errático de resposta sinaliza que se deve dar mais atenção na interpretação do escore de indivíduos com hemiparesia nesse item, sendo importante tentar identificar o que pode ter contribuído para o comportamento inesperado observado ${ }^{12,15}$. Em relação ao uso do BESTest em hemiparéticos, recomenda-se observar se os fatores associados aos comportamentos inesperados estáo relacionados ao equilíbrio ou a ausência de movimentação comumente encontrada nesses indivíduos.

A análise fatorial evidenciou que ambos os instrumentos foram multidimensionais. $\mathrm{O}$ equilíbrio é um construto amplo que envolve vários sistemas ${ }^{1}$. Como o BESTest é dividido em seçóes, pode-se considerar que o instrumento foi desenvolvido com o objetivo de abranger vários aspectos relacionados ao equilíbrio, ou seja, a partir de um modelo multidimensional. Dessa forma, os resultados indicativos da multidimensionalidade confirmam a sua função original. Porém, a divisão dos itens segundo os resultados da análise de componente principal, não seguiu o modelo original, agrupando itens de diferentes seções sem significado teórico evidente. Esperava-se que o MiniBESTest fosse unidimensional, como em sua versão original, já que o instrumento foi construído a partir do construto "equilíbrio dinâmico"7. Entretanto, o instrumento também foi multidimensional e a análise evidenciou que alguns itens poderiam ser removidos sem grande prejuízo para as qualidades psicométricas. Com exceção de passar de sentado para de pé, todos os itens que poderiam ser removidos são da escala de respostas posturais reativas. Entretanto, a exclusão de itens em uma escala já existente deve ser o último recurso a ser considerado porque os itens podem ser importantes clinicamen$t^{29}$. No presente estudo, se os itens fossem removidos, não seriam avaliadas as respostas posturais reativas, que são importantes para avaliar como o indivíduo reage após uma perturbação do equilíbrio ${ }^{1}$. Dessa forma, tanto ques- 
tốes psicométricas quanto clinicas devem ser consideradas, não sendo recomendada exclusão de nenhum item. Outro ponto a ser considerado é que, dado o tamanho da amostra, pequenas flutuações no padrão de pacientes individuais podem ter influenciado o resultado global.

O MiniBESTest, quando comparado ao BESTest, se adequou melhor ao modelo, devido a ausência de itens erráticos. Por outro lado, sendo o BESTest um instrumento voltado para diagnóstico dos déficits de equilíbrio, a análise Rasch mostrou que seus itens estão menos espaçados e cobrem uma maior porção do contínuo de habilidade. Em um instrumento voltado para triagem como o MiniBESTest, a calibração dos itens é mais espaçada, o que implica em menor precisão das medidas.

Uma limitação para o uso do BESTest na prática clínica é o tempo despendido para sua aplicação. Estudos anteriores relataram tempo de aplicação variando de 20 a 31 minutos $^{1,10}$ até 45 a 60 minutos ${ }^{9}$. Neste estudo, o tempo gasto foi de 30 a 45 minutos. Uma possibilidade seria a aplicação das seçóes separadamente ${ }^{1}$. Os dados do presente estudo, no entanto, não dão suporte à divisão das seçôes como proposta no teste. Assim, são necessários novos estudos que avaliem a validade e a utilidade clínica das seçốes quando utilizadas de forma independente nessa população.

Uma limitação deste estudo foi a amostra restrita a hemiparéticos crônicos. Seriam interessantes novos estudos incluindo indivíduos em fase aguda e subaguda após AVC com diferentes níveis de habilidade. São importantes, também, novos estudos que avaliem outras propriedades psicométricas destes instrumentos em indivíduos com hemiparesia, como responsividade e validade de critério preditiva. Como o BESTest apresenta a proposta de direcionar o tratamento na prática clínical, são também necessários estudos controlados e randomizados para verificar se o tratamento direcionado às seçóes do BESTest será realmente mais eficaz.

\section{CONCLUSÃO}

As versôes brasileiras do BESTest e do MiniBESTest mostraram ser confiáveis e capazes de discriminar diferentes níveis de habilidade entre os indivíduos, sem ser observado efeito teto ou solo.
De uma forma geral, a hierarquia dos itens e adequação ao modelo Rasch forneceu evidência quanto à validade de construto dos instrumentos. Recomenda-se, porém, cautela na interpretação do escore total do BESTest e atenção em relação aos itens identificados como erráticos. Dado que a multidimensionalidade náo impede o uso de um instrumento como ferramenta clinicamente válida ${ }^{30}$, os dois instrumentos parecem ser úteis para a avaliação do equilíbrio em indivíduos com hemiparesia crônica.

\section{REFERÊNCIAS}

1.Horak FB, Wrisley DM, Frank J. The Balance Evaluation Systems Test (BESTest) to differentiate balance deficits. Phys Ther 2009;89:484-98. http://dx.doi.org/10.2522/ptj.20080071

2.Mancini M, Horak FB. The relevance of clinical balance assessment tools to diferenciate balance deficits. Eur J Phys Rehabil Med 2010;46:239-48.

3.Campbell GB, Matthews JT. An integrative review of factors associated with falls during post-stroke rehabilitation. J Nurs Scholarsh 2010;42:395-404. http://dx.doi.org/10.1111/j.1547-5069.2010.01369.x

4.Schmid AA, Rittman M. Consequences of poststroke falls: activity limitation, increased dependence, and the development of fear of falling. Am J Occup Ther 2009;63:310-6. http://dx.doi.org/10.5014/ajot.63.3.310

5.Fryberg GE, Lindmark B, Lanshammar H, Borg J. Correlation between clinicalassesment and force plate measurement of postural control after stroke. J Rehabil Med 2007;39:448-53. http://dx.doi.org/10.2340/16501977-0071 6.Blum L, Korner-Bitensky N. Usefulness of the Berg Balance Scale in stroke rehabilitation: A sistematic review. Phys Ther 2008;88:559-66. http://dx.doi. org/10.2522/ptj.20070205

7.Franchignoni F, Horak F, Godi M, Nardone A, Giordano A. Using psychometric techniques to improve the Balance Evaluation Systems Test: the mini-BESTest. J Rehabil Med 2010;42:323-31. http://dx.doi.org/10.2340/16501977$\underline{0537}$

8.Leddy AL, Crowner BE, Earhart GM. Utility of the Mini-BESTest, BESTest, and BESTest sections for balance assessments in individuals with Parkinson disease. J Neurol Phys Ther 2011;35:90-7. http://dx.doi.org/ 10.1097/ NPT.0b013e31821a620c

9.Parminder K, Padgett JV, Jacobs, SLK. Is the BESTest at its best? A suggested briefversion based on interrater reliability, validity, internal consistency, and theoretical construct. Phys Ther 2012;92:1197-207. http://dx.doi. org/10.2522/ptj.20120056

10.Maia AC, Rodrigues-de-Paula F, Magalhães LC, Teixeira RL. Cross-cultural adaptation and analysis of psychometric properties of the Balance Evaluation Systems Test and MiniBESTest in the elderly and individuals with Parkinson's disease: application of the Rasch model. Braz J Phys Ther 2013;17:195-217. http://dx.doi.org/10.1590/S1413-35552012005000085

11.Portney LG, Watkins MP. Foundations of Clinical Research: Applications to Practice. 2ed. New Jersey: Prentice Hall Health, 2000, 742p.

12.Velozo CA, Forsyth K, Kielhofner G. Objective measurement: the influence of item response theory on research and practice. In: Kielhofner G. Research in Occupational Therapy: Methods of Inquiry for enhancing Practice. Philadelphia: F. A. Davis Company, 2006, p.177-200. 
13.Linacre JM. Sample size and item calibration stability. Rasch Meas Trans 1994;7:328.

14.Linacre JM. Optimizing rating scale category effectiveness. J Appl Meas 2002;3:85-106.

15.Saliba VA, Faria CD, Laurentino GE, Cassiano JG, Teixeira-Salmela LF. Adaptaçáo transcultural e análise das propriedades psicométricas da versão brasileira do instrumento Motor Activity Log. Rev Panam Salud Pub 2011;30:262-71. http://dx.doi.org/10.1590/S1020-49892011000900011

16.Neckel N, Pelliccio M, Nichols D, Hidler J. Quantification of functional weakness and abnormal synergy patterns in the lower limb of individuals with chronic stroke. J Neuroeng Rehabil 2006;3:1-11. http://dx.doi. org/10.1186/1743-0003-3-17

17.Teixeira-Salmela LF, Devaraj R, Olney SJ. Validation of the human activity profile instroke: a comparison of observed, proxy and self-reported scores. Disabil Rehabil 2007;29:1518-24. http://dx.doi.org/10.1080/09638280601055733

18.Centers for Disease Control and Prevention. Physical activity trends - United States, 1990 -1998. Morbidity and Mortality Weekly Report (MMWR) 2001;50:166-9.

19.Michaelsen SM, Rocha AS, Knabben RJ, Rodrigues LP, Fernandes CGC. Tradução, adaptação e confiabilidade interexaminadores do manual de administração da escala de Fugl-Meyer. Braz J Phys Ther 2011;15:80-8.

20.Sim J, Wright CC. The kappa statistic in reliability studies: use, interpretation, and sample size requirements. Phys Ther 2005;85:257-68.

21.Linacre JM, Wright BD (Endereço na Internet). Winsteps Rasch Measurement Version 3.74.0 (Software). Disponível em: http://www. winsteps.com 22.Chen HF, Wu CY, Lin KC, Chen HC, Chen CP, Chen CK. Rasch validation of the Streamlined Wolf Motor Function Test in people with chronic stroke and subacute stroke. Phys Ther 2012;92:1017-26. http://dx.doi. org/10.2522/ptj.20110175

23.Leddy AL, Crowner BE, Earhart GM. Functional gait assesment and Balance Evaluation System Test: Reliability, validity, sensitivity, and specificity for identifying individuals with Parkinson Disease who fall. Phys Ther 2011;91:102-12. http://dx.doi.org/10.2522/ptj.20100113

24.Jones KD, Horak FB, Winters KS, Morea JM, Bennett RM. Fibromyalgia is associated with impaired balance and falls. J Clin Rheumatol 2009;15:1621. http://dx.doi.org/10.1097/RHU.0b013e318190f991

25.Jacobs JV, Kasser SL. Balance impairment in people with multiple sclerosis: Preliminary evidence for the Balance Evaluation Systems Test. Gait Post 2012;36:414-8. http://dx.doi.org/10.1016/j.gaitpost.2012.03.026

26. Banerjee A, Jadhav SL, Bhawalkar JS. Limitations of activities in patients with musculoskeletal disorders. Ann Med Health Sci Res 2012;2:5-9.

27.Mansfield A, Inness EL, Lakhani B, McIlroy WE. Determinants of limb for preference initiating compensatory stepping poststroke. Arch Phys Med Rehabil 2012;93:1179-84. http://dx.doi.org/10.1016/j.apmr.2012.02.006

28.Genthon N, Rougier P, Gissot A, Froger J, Pélissier J, Pérennou D. Contribution of each lower limb to upright standing in stroke patients. Stroke 2008;39:1793-9. http://dx.doi.org/10.1161/STROKEAHA.107.497701

29.Nilsson AL, Tennant A. Past and present issues in Rasch analysis: the functional independence measure (FIM) revisited. J Rehabil Med 2011;43; 88491. http://dx.doi.org/10.2340/16501977-0871

30.Lovaglio PG, Monzani EM. Validation aspects of the health of the nation outcome scales. Inter J Mental Health Sys 2011;5:1-11. http://dx.doi. org/10.1186/1752-4458-5-20 